\title{
Transcriptomic Analysis Reveals Candidate Genes Responsive to Sclerotinia scleroterum and Cloning of the Ss-Inducible Chitinase Genes in Morus laevigata
}

\author{
Huanhuan Jiang 1,2,3,4,5,+, Xiaoyun Jin 1,2,3,4,5,+, Xiaofeng Shi 1,2,3,4,5, + , Yufei Xue 1,2,3,4,5, \\ Jiayi Jiang 1,2,3,4,5, Chenglong Yuan 1,2,3,4,5, Youjie Du 1,2,3,4,5, Xiaodan Liu 1,2,3,4,5, \\ Ruifang Xie 1,2,3,4,5, Xuemei Liu 1,2,3,4,5, Lejing Li 1,2,3,4,5, Lijuan Wei 1,2,3,4,5, \\ Chunxing Zhang ${ }^{1,2,3,4,5}$, Liangjing Tong $1,2,3,4,5$ and Yourong Chai $1,2,3,4,5, * \mathbb{D}$ \\ 1 College of Agronomy and Biotechnology, Southwest University, Chongqing 400715, China; \\ jh8469259@email.swu.edu.cn (H.J.); jxy0606@163.com (X.J.); kamiyahiko@163.com (X.S.); \\ xyf710@swu.edu.cn (Y.X.); jjy326@email.swu.edu.cn (J.J.); ycl416@email.swu.edu.cn (C.Y.); \\ ad1994@email.swu.edu.cn (Y.D.); liu1500@email.swu.edu.cn (X.L.); xrf2019@email.swu.edu.cn (R.X.); \\ lxm804527660@email.swu.edu.cn (X.L.); lilejing123@email.swu.edu.cn (L.L.); lijuanwei@swu.edu.cn (L.W.); \\ wjmandzcx13@163.com (C.Z.); tlj6793@163.com (L.T.) \\ 2 Academy of Agricultural Sciences, Southwest University, Chongqing 400715, China \\ 3 Chongqing Engineering Research Center for Rapeseed, Southwest University, Chongqing 400715, China \\ 4 Chongqing Key Laboratory of Crop Quality Improvement, Southwest University, Chongqing 400715, China \\ 5 Engineering Research Center of South Upland Agriculture of Ministry of Education, Southwest University, \\ Chongqing 400715, China \\ * Correspondence: chaiyour@swu.edu.cn; Tel.: +86-13527547178 \\ + These authors contributed equally to this work.
}

Received: 22 September 2020; Accepted: 3 November 2020; Published: 7 November 2020

\begin{abstract}
Sclerotinia sclerotiorum (Ss) is a devastating fungal pathogen that causes Sclerotinia stem rot in rapeseed (Brassica napus), and is also detrimental to mulberry and many other crops. A wild mulberry germplasm, Morus laevigata, showed high resistance to $S s$, but the molecular basis for the resistance is largely unknown. Here, the transcriptome response characteristics of $M$. laevigata to Ss infection were revealed by RNA-seq. A total of 833 differentially expressed genes (DEGs) were detected after the $S$ s inoculation in the leaf of M. laevigata. After the GO terms and KEGG pathways enrichment analyses, 42 resistance-related genes were selected as core candidates from the upregulated DEGs. Their expression patterns were detected in the roots, stems, leaves, flowers, and fruits of M. laevigata. Most of them (30/42) were specifically or mainly expressed in flowers, which was consistent with the fact that $S$ s mainly infects plants through floral organs, and indicated that Ss-resistance genes could be induced by pathogen inoculation on ectopic organs. After the Ss inoculation, these candidate genes were also induced in the two susceptible varieties of mulberry, but the responses of most of them were much slower with lower extents. Based on the expression patterns and functional annotation of the 42 candidate genes, we cloned the full-length gDNA and cDNA sequences of the Ss-inducible chitinase gene set (MlChi family). Phylogenetic tree construction, protein interaction network prediction, and gene expression analysis revealed their special roles in response to $S s$ infection. In prokaryotic expression, their protein products were all in the form of an inclusion body. Our results will help in the understanding of the molecular basis of Ss-resistance in M. laevigata, and the isolated MlChi genes are candidates for the improvement in plant Ss-resistance via biotechnology.
\end{abstract}

Keywords: Sclerotinia sclerotiorum; Sclerotinia stem rot; mulberry; Morus laevigata; chitinase; RNA-seq 


\section{Introduction}

Sclerotinia sclerotiorum (Ss) is a significant fungal pathogen that causes Sclerotinia stem rot (SSR) in rapeseed (Brassica napus L.) [1,2]. Except for the rapeseed, as a nonhost-specific, omnivorous, and necrotrophic plant pathogen, Ss can infect more than 400 plant species around the world, and result in a significant yield and quality reduction in many important crops [3-5]. In China, the incidence of SSR in the rapeseed growing areas is about $10 \%$ to $80 \%$, and yield loss of diseased plants ranges from $10 \%$ to $73 \%$. Therefore, it is urgent to breed Ss-resistant rapeseed varieties. However, there is currently a lack of highly $S$ s-resistant breeding germplasm in rapeseed. In addition, although some researchers have explored the interaction relationships between various crops and $S s$ in multiple aspects [6-10], the genetic and molecular basis of the interactions of $S s$ with its host crops is still poorly understood.

Mulberry (Morus sp.) is a kind of deciduous tree, the leaves of which can be used as food for rearing silkworm (Bombyx mori), and its fruits not only have a delicious taste but are also health-promoting because of the rich nutraceuticals such as anthocyanin [11,12]. In order to encourage the leaves growth that can feed silkworms, the mulberry plants need to be trimmed regularly, which makes it susceptible to pests and pathogens [13]. Similar to rapeseed, the mulberry plants are also susceptible to sclerotiose [14]. Mulberry fruit sclerotiniosis is also called white fruit disease, which includes mulberry sorosus hypertrophic sclerote disease, mulberry sorosus parvulling sclerote disease, and mulberry sorosus diminuting sclerote disease caused by Ciboria shiraiana, Ciboria carunculoides, and Scleromitrula shiraiana, respectively $[14,15]$. Once the mulberry is infected with the pathogen, the fruit turns gray and begins to rot, which seriously affects its economic value [14]. Recently, several researchers also isolated the $S s$ from the diseased mulberry fruit $[16,17]$. After artificial inoculation with the ascospore of Ciboria shiraiana in rapeseed, the infected plants showed typical characteristics of SSR. With the artificial inoculation of $S s$ in mulberry, the morphogenesis symptoms of the infected fruit were consistent with the mulberry sorosus hypertrophic sclerote disease [18]. These results indicate that the pathogen of mulberry sorosus hypertrophic sclerote disease and the $S s$ in rapeseed can cross-infect each other. Interestingly, the natural sclerotiose disease of mulberry is more frequently caused by the other three pathogens than by $S s$, though it is fatal to rapeseed. That is to say, between the two hosts, $S$ s prefers rapeseed over mulberry, or mulberry is more resistant than rapeseed to $S s$ naturally. Different mulberry stocks vary distinctly in their susceptibility to sclerotiose pathogens. A wild germplasm of the mulberry, Morus laevigata (Himalyan mulberry or "Long-fruit mulberry"), showed resistance to $S$ s, which means that it can be used as a resistant germplasm resource $[19,20]$. Therefore, a better understanding of the resistance mechanism of mulberry, especially of $M$. laevigata to $S s$, is not only useful for digging out the resistance-related genes, but also significant for breeding $S$ s-resistant rapeseed varieties via biotechnology. Though all four pathogens (C. shiraiana, C. carunculoides, S. shiraiana, and S. sclerotiorum) can infect mulberry, S. sclerotiorum is not the major type. In rapeseed, however, S. sclerotiorum is the major type of pathogen, while the first three are absolutely minor ones. Therefore, we assume that the exchange of the major resistance genes between mulberry and rapeseed should be helpful to each other to enhance resistance to white rot disease. As M. laevigata is the most Ss-resistant stock in the genus Morus, we believe that the major Ss-inducible resistance genes cloned from M. laevigata will be valuable for molecular breeding of the $S s$-resistance of $S$ s-prevalent crops such as rapeseed, sunflower, and soybean, and might be effective in enhancing white rot disease of mulberry itself too.

Pathogen-associated molecular pattern (PAMP)-triggered immunity (PTI) and effector-triggered immunity (ETI) are the two main reaction processes of the plants' innate immune systems against the infection of pathogens [21]. However, the steps for the host plant immune response against $S$ s remain unclear. Analysis of the genome sequences of $S s$ indicated that its genome contains a large number of genes that encode secretory effector proteins that may be involved in the interaction between $S s$ and the host [22]. Though recent studies have shown that certain types of secreted proteins are important pathogenic factors, only a few studies have actually identified a host receptor of the $S$ s effectors [23]. For example, the small secreted protein SsSSVP1 was secreted from the hyphae and then located in the cytoplasm of the host, which could interact with the QCR8 protein of the host, resulting in changes in 
the subcellular localization of QCR8, and induce significant plant cell death [24]. Another secreted protein SsCP1 could interact with the PR1 protein of the host in the apoplast to facilitate infection of $S s$, and over-expression of SsCP1 in Nicotiana benthamiana could significantly induce cell death, while over-expression of $P R 1$ enhanced the resistance to $S s$ [25]. These results indicate that the potential effectors play important roles in the host-pathogen interactions during the infection of $S s$ to plants.

Chitin is another kind of effector, which is the major component of the cell walls of fungi, but not in plants [26]. However, chitinase is widely present in plant cells. When the plants are infected by pathogens, the activities of chitinase increase rapidly. It can catalyze the hydrolysis of $\beta-1,4$ glycoside bond linkages of chitin polymers of the fungal cell wall into $\mathrm{N}$-acetyl glucosamine oligomers and monomer components, resulting in cell wall degradation and death of pathogens [22,26]. Hence, chitinase is also considered as the pathogenesis-related (PR) proteins in plants and has been widely used in genetic improvement of the disease resistance of crops by genetic engineering [27,28]. In mulberry, twenty chitinase genes (Mnchi1-Mnchi20) were identified from the M. notabilis genome, and some of them were confirmed to respond to insect wounding and fungal infection [29]. Heterologous expression of mulberry latex chitinase gene MaMLX-Q1 in Arabidopsis enhanced the defense against Plutella xylostella of the transgenic plants [30]. In rapeseed, co-expression of a defensin gene from Raphanus sativus and a chimeric chitinase gene chit42 from Trichoderma atroviride enhanced the resistance against SSR [31]. Additionally, co-expression of the chit42 gene mentioned above and a polygalacturonase-inhibiting protein 2 gene (PG1P2) from Phaseolusvulgaris also showed increased resistance to SSR in transgenic rapeseed [32]. Therefore, the isolation of Ss-inducible chitinase genes from $S s$-resistant mulberry cultivars has potential application value in breeding $S$ s-resistant rapeseed cultivars via genetic engineering.

To date, there is no report on the systemic identification of the Ss-responsive molecular mechanism and systemic cloning of $S s$-responsive Chi genes from mulberry species. In this study, the transcriptomic changes of the M. laevigata leaves before and after Ss-inoculation were analyzed to identify the candidate genes in response to $S s$. Then, the expression patterns of the selected candidate genes among different tissues and varieties of mulberry were analyzed to further verify their roles. Additionally, as functional annotation showed that chitinase genes constitute a core set of the Ss-responsive defensive genes, we isolated their full-length cDNAs and corresponding genomic sequences (gDNAs) from M. laevigata, and the molecular features of the genes and their encoded proteins were analyzed bioinformatically. Our results provide a basis for understanding the Ss-resistance mechanism of M. laevigata, and the Ss-inducible chitinase genes isolated in this study have potential application in the genetic improvement of $S$ s-resistance in crops such as rapeseed, sunflower, and soybean, which are highly susceptible to $S s$.

\section{Results}

\subsection{RNA-seq and De Novo Transcriptome Assembly}

Two RNA-seq libraries (ML_SS0 and ML_SS1) were constructed in this study. Then, they were subjected to paired-end RNA sequencing using the Illumina HiSeq2500 platform, and on average, 25.98 million and 25.02 million clean reads were obtained from the two libraries, respectively (Table S1). Due to the lack of information about the M. laevigata genome, these clean reads were de novo assembled using the Trinity software [33]. Finally, a total of 91,166 transcripts and 61,985 unigenes were assembled, and the mean length of the unigenes was $784 \mathrm{bp}$ and the N50 was $1487 \mathrm{bp}$ (Table S1, Figure 1A). 
A

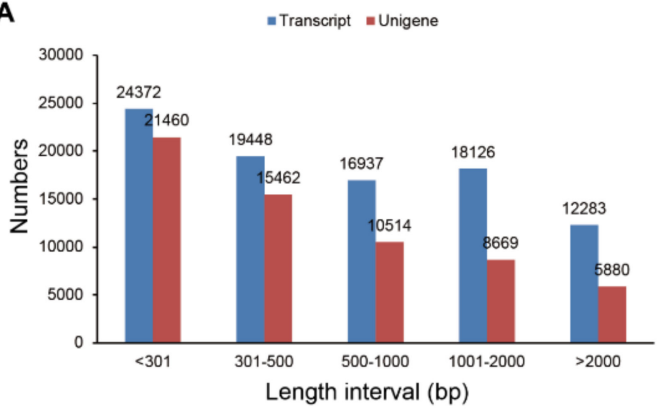

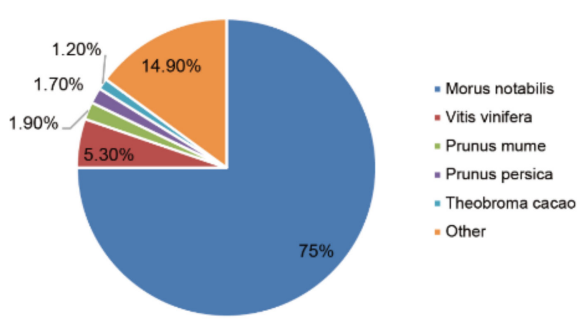

Figure 1. De novo transcriptome assembly of $M$. laevigata. (A) The length distribution of the M. laevigata transcripts and unigenes; (B) species distribution of the unigene BLAST results against the Nr database with the cutoff E-value of $10^{-5}$.

\subsection{Functional Annotation of the Unigenes}

All of the unigenes were annotated using the BLAST algorithm in seven public databases with the E-value of $10^{-5}$. The results showed that 27,991 (45.15\%), 14,341 (23.13\%), $7258(11.7 \%), 18,506(29.85 \%)$, 18,463 (29.78\%), 19,030 (30.7\%), and 9779 (15.77\%) unigenes were annotated in Nr, Nt, KEGG, Swiss-Prot, PFAM, GO, and KOG databases, respectively (Table 1). A total of 3804 unigenes were annotated in all of the seven databases, and 30,334 (48.93\%) unigenes were annotated in at least one database. The unigenes of $M$. laevigata showed the best matches with M. notabilis $(75.0 \%)$, followed by Vitis vinifera (5.3\%), Prunus mume (1.9\%), Prunus persica (1.7\%), and Theobroma cacao (1.2\%) (Figure 1B).

Table 1. The statistical analysis of the annotated unigenes.

\begin{tabular}{ccc}
\hline Databases & Annotated Unigenes & Percentage (\%) \\
\hline $\mathrm{Nr}$ & 27,991 & 45.15 \\
$\mathrm{Nt}$ & 14,341 & 23.13 \\
KEGG & 7258 & 11.71 \\
Swiss-Prot & 18,506 & 29.85 \\
Pfam & 18,463 & 29.78 \\
GO & 19,030 & 30.70 \\
KOG & 9779 & 15.77 \\
Annotated in all Databases & 3804 & 6.13 \\
Annotated in at least one Database & 30,334 & 48.93 \\
Total Unigenes & 61,985 & 100.00 \\
\hline
\end{tabular}

\subsection{Analysis of Differentially Expressed Genes}

By comparing the two libraries, a total of 47,029 common unigenes were identified in both of them (Figure 2A). The expression data of each gene were normalized using FPKM, and a total of 833 differentially expressed genes (DEGs) (624 upregulated and 209 downregulated in ML_SS1) were detected by the DESeq method with the threshold of adjusted $p$-value $<0.05$ and $\log 2$ (fold change) $\mid \geq 1$ (Figure 2B, Table S2). Then, the DEGs were enriched in the GO and KEGG database. For the GO enrichment, the majority of the DEGs were enriched in the biological process (BP) and molecular function (MF) GO categories. The GO terms oxidation-reduction process (GO:0055114), single-organism metabolic process (GO:0044710), and metabolic process (GO:0008152) were mostly enriched in the BP category, and oxidoreductase activity (GO:0016491), endopeptidase inhibitor activity (GO:0004866), and endopeptidase regulator activity (GO:0061135) were the most significantly enriched GO terms in the MF category (Table S3). For the KEGG analysis, the 833 DEGs were enriched in 217 KEGG pathways (Table S4), and the 20 pathways with the highest enrichment are shown in Figure 3. Among the top 20 pathways, carbon fixation in photosynthetic organisms, galactose metabolism, 
glycolysis/gluconeogenesis, nitrogen metabolism, and carbon metabolism were significantly enriched, which include 14, 9, 14, 7, and 22 DEGs, respectively.

A

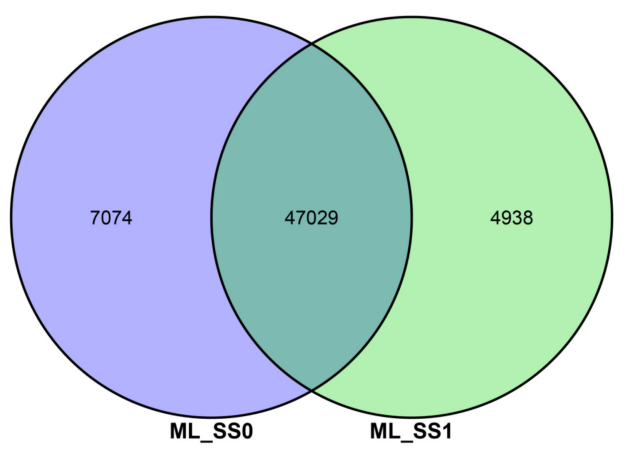

B

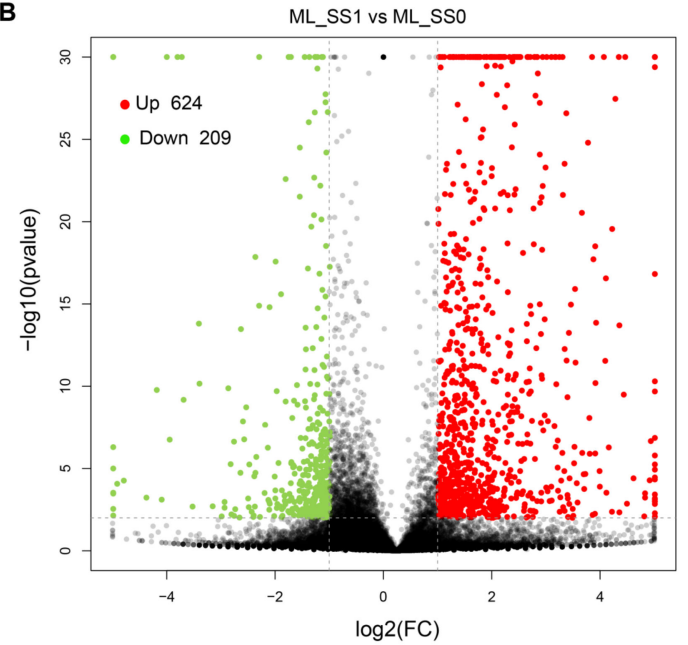

Figure 2. Screening of differentially expressed genes (DEGs). (A) Venn chart of co-expressed genes between the samples. (B) Numbers of up- and down-regulated DEGs in the infected leaves of M. laevigata.

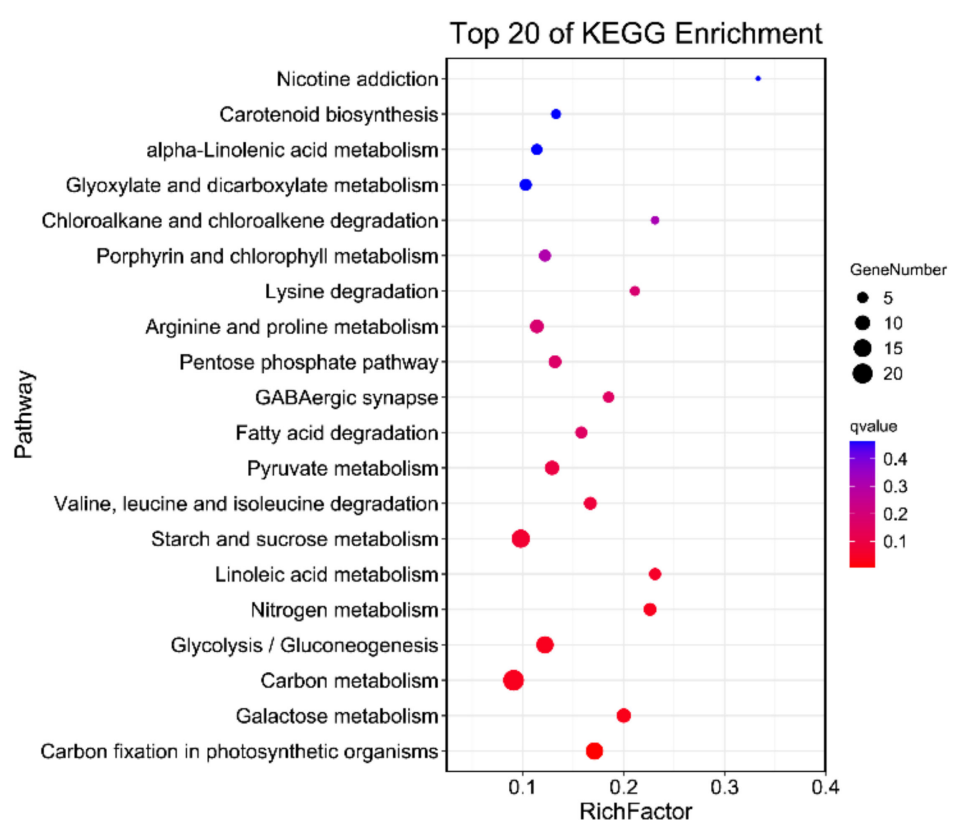

Figure 3. The top 20 statistics of KEGG pathway enrichment for the DEGs. The Rich factor is the ratio of DEGs in this pathway term to all the number of genes annotated in this pathway term.

\subsection{Candidate Genes Screening}

According to the biological pathway description, several pathways that were closely related to disease resistance processes had been selected, such as the oxidation-reduction process, protein phosphorylation and proteolysis, metabolic process, and transcription factor and chitin metabolic process. Based on the expression levels of the DEGs in these biological pathways, a total of 42 upregulated DEGs were selected as the candidate genes in response to the infection of S. sclerotiorum (Table 2). These candidate genes were involved in endopeptidases, oxidoreductases, glucose metabolism, nitrogen metabolism, pathogenesis-related proteins, and transcription factors. Their expression levels were also validated using quantitative real-time (qRT)-PCR, which showed 
that the expression trend of 38 genes was consistent with that of RNA-seq, and only four genes (c30732_g1, c35494_g1, c40703_g1, and c45307_g1) had an inconsistent expression trend (Figure S2), which may be caused by the difference between the two detection platforms. For the above 38 genes, Pearson's correlation was used to detect their expression correlation under the two platforms in SPSS software, and the results showed that the correlation between them reached a significant level $(\mathrm{r}=0.459$, $\mathrm{n}=38, p=0.004)$ (Figure 4).

Table 2. The 42 candidate genes that were screened from the differentially expressed genes.

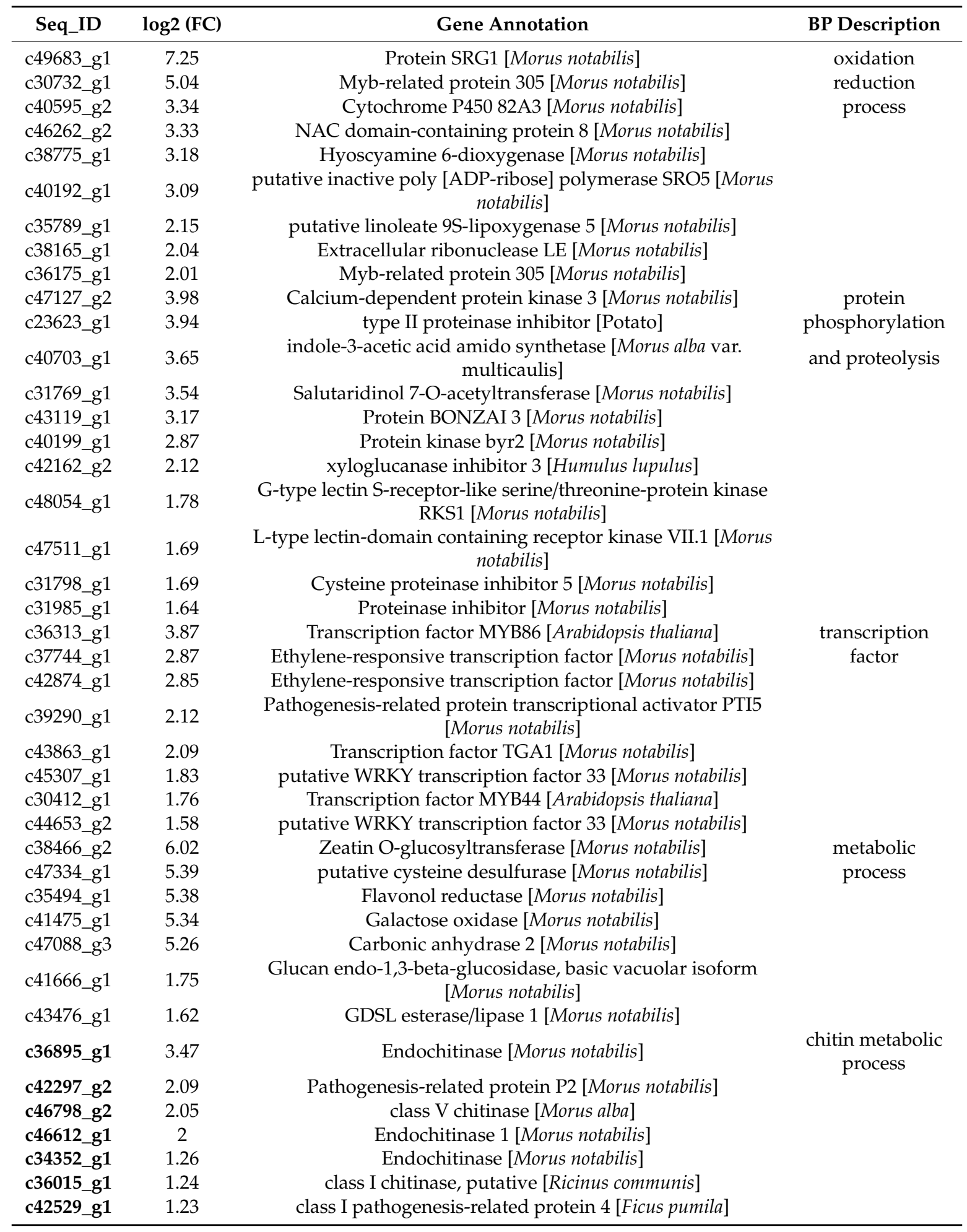




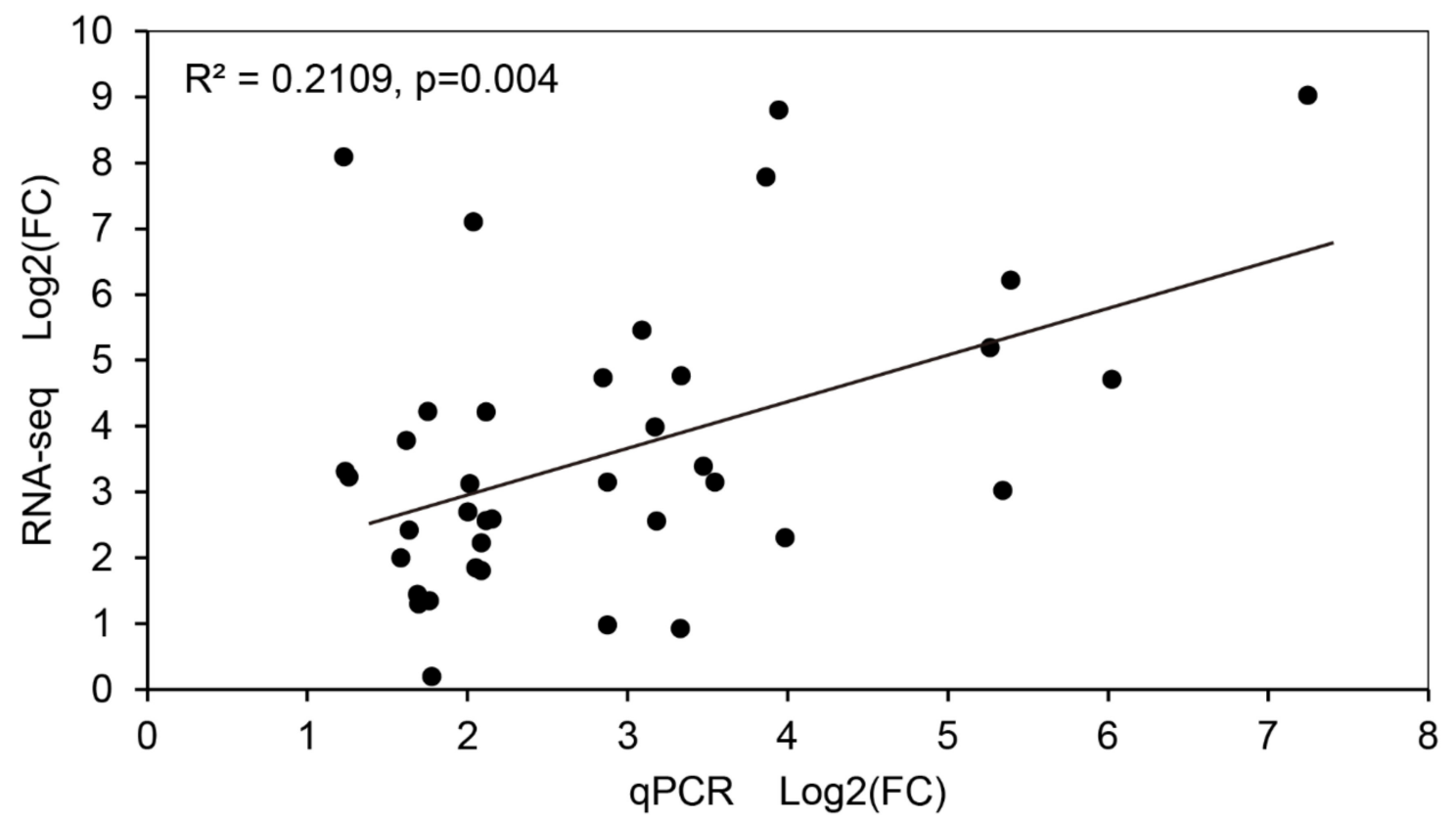

Figure 4. Correlation analysis of the gene expression fold change (FC) calculated from the quantitative real-time (qRT)-PCR and RNA-seq data. Pearson's correlation (two tailed) was used for estimating $\mathrm{p}$-values and $\mathrm{r}(\mathrm{n}=38)$ in SPSS 20.0.

\subsection{Organ-Specificity of Transription Patterns of the Candidate Genes}

The expression patterns of the 42 candidate genes were detected in the roots, stems, leaves, flowers, and fruits of the Ml using qRT-PCR, and most of them showed tissue-specific expression patterns (Figure 5). For example, c30732 was specially expressed in roots, $c 46262$ and $c 42874$ were mainly expressed in stems, c36895, c42297, c36015, and c42529 showed a high expression in leaves, and c38165, $c 49683, c 47088$, and $c 47127$ were predominantly expressed in fruits. Interestingly, the majority of the candidate genes (30/42) were specially or mainly expressed in flowers. 


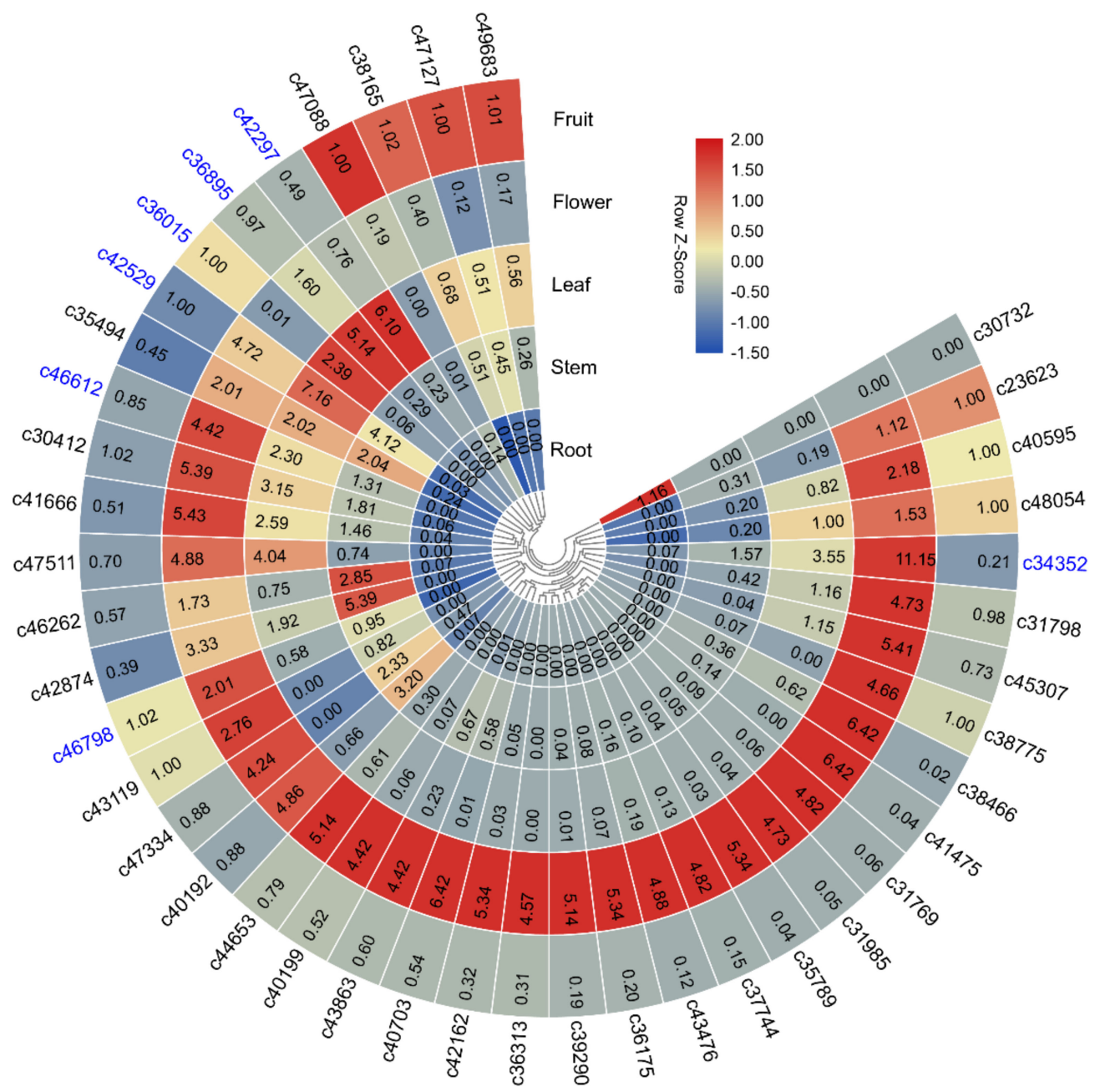

Figure 5. The expression patterns of the 42 candidate genes in different tissues of M. laevigata. The gene expression patterns were examined by qRT-PCR, and were visualized using the TBtools toolkit. The values in the heat map represent the relative expression levels of the genes. The expression patterns represented by the bar charts are shown in Figure S1.

\subsection{Stock-Diferred Expression and Ss-Inducibility Dynamics of the Candidate Genes}

The expression patterns of the 42 candidate genes in response to the infection of S. sclerotiorum in different resistance stocks of mulberry were also detected using qRT-PCR. First, the expression levels of the candidate genes in the mixed samples of three cultivars at different periods after infection were analyzed (Figure 6A). As expected, almost all of them were upregulated in the three varieties after infection, but the extent to which they were expressed varied among resistance stocks. For example, c45307, c23623, c34352, c47334, c36895, c43476, c38165, c40192, c37744, c49683, c30412, c46798, c47127, $c 47511, c 44653, c 31798, c 42874, c 41666$, and $c 42162$ were highly induced in Ml (high resistance to Ss), while $c 46262, c 40595, c 47088, c 40703, c 40199, c 41475, c 35494, c 35789, c 36313, c 38466, c 42297$, and $c 43119$ were mainly induced in JL (partial resistance to $S s$ ). However, their induction intensity in Ma (low resistance to $\mathrm{Ss}$ ) was lower than those in $\mathrm{Ml}$ and JL.

Then, the expressions of the 42 candidate genes in different infection periods of the three resistance stocks were detected. As shown in Figure 6B, the majority genes were significantly induced in Ml with peak expression at $3 \mathrm{~h}$ after infection. More than half of them also showed certain inducibility in JL or in $\mathrm{Ma}$, but with peaking expression much later than in $\mathrm{Ml}$ (e.g., $48 \mathrm{~h}$ after infection). 
A

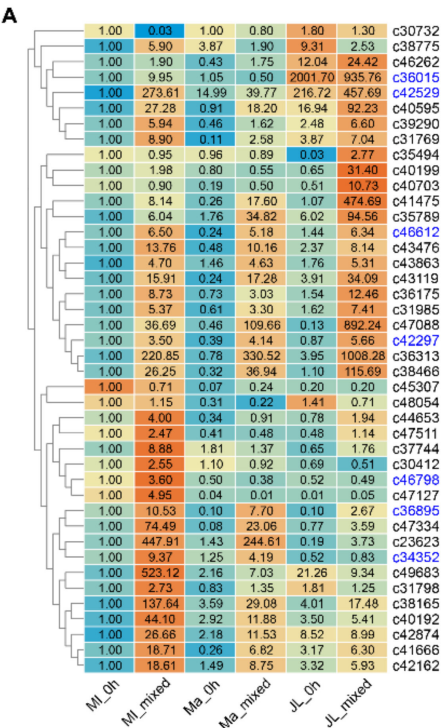

B

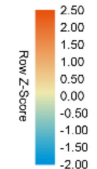

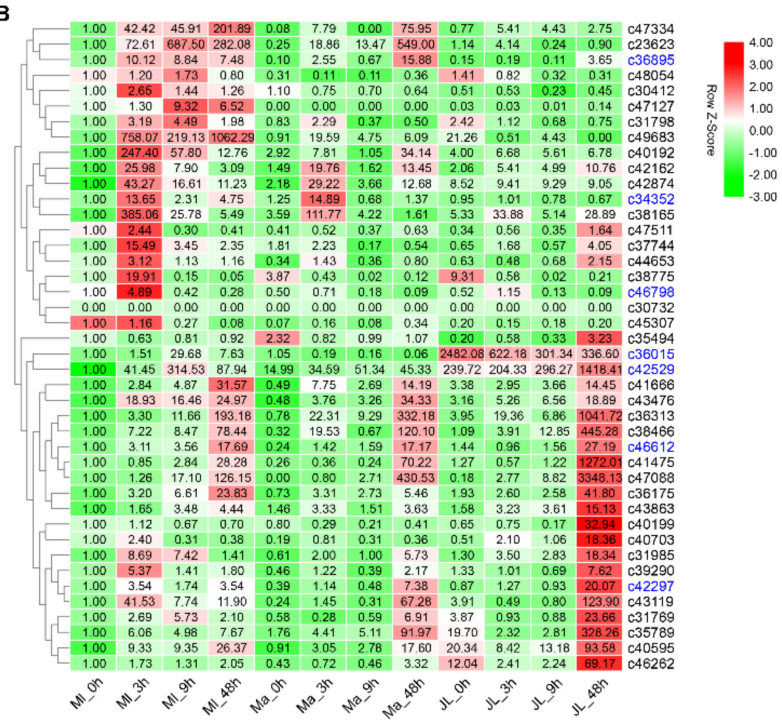

Figure 6. The expression patterns of the 42 candidate genes in different varieties of mulberry. (A) The expression patterns of the 42 candidate genes in the mixed samples at different stages after infection in different varieties. (B) The expression patterns of the 42 candidate genes at different stages after infection in different varieties. The gene expression analysis was examined by qRT-PCR, and all of the qRT-PCR values were expressed relative to the expression level of Ml_Oh. The expression patterns represented by the bar charts are shown in Figures S2 and S3.

\subsection{Cloning and Molecular Features of the Ss-Inducible Chitinase Genes from M. Laevigata}

Among the 42 candidate genes, according to the functional annotation, unigenes $c 36895, c 42297$, $c 46612, c 46798, c 34352, c 36015$, and $c 42529$ were chitinase genes. Based on the homology with chitinase genes in other species, they were renamed as MlChiIV, MlHEL, MlChiIA, MlChiV, MlChiIB, MlChiIC, and MlChiID, respectively. Then, the full-length cDNA and corresponding gDNA sequences of the seven chitinase genes were isolated from $M$. laevigata. Finally, a total of fifteen homologous sequences were obtained and used for subsequent analysis. In sequence alignment, MlChiIC1 was very similar to MlChiIC2, while MlChiV2-1 and MlChiV2-2 were very similar to MlChiV1-2 and MlChiV1-2, respectively, which might be cloned from heterozygous allelic pairs. Due to the very high homology, their cloning primers were identical to each other, and could not be cloned by separate PCRs. In one-tube PCR gDNA-amplification for the highly homologous genes, the preferred amplification of one allele and suppressed amplification of another allele was a common phenomenon. We repeated the cloning process several times, and only gDNAs of MlChiIC2, MlChiV1-2, and MlChiV1-2 were obtained, while gDNAs of MlChiIC1, MlChiV2-1, and MlChiV2-2 were not obtained yet.

As shown in Table 3, except that MlChiIC1, MlChiV2-1, and MlChiV2-2 did not obtain gDNA sequences, the length of gDNA and cDNA of the fifteen genes ranged from $1284 \mathrm{bp}$ (MlHEL2) to $3603 \mathrm{bp}$ (MlChiV1-2) and $753 \mathrm{bp}$ (MlHEL) to $1332 \mathrm{bp}$ (MlChiIV2), respectively. The numbers of amino acid (aa) residues of encoded proteins ranged from 144 (MlHEL) to 390 (MlChiV2), the relative molecular weight varied from 15.75 to $43.57 \mathrm{kDa}$, and their isoelectric point $(p I)$ ranged from 4.62 to 6.84. The GRAVY of them varied from -0.42 to -0.20 , showing that they were all hydrophilic proteins. Additionally, the signal peptide prediction showed that they all had signal peptides. Conserved domain analysis showed that MlChiIA, MlChiIB, and MlChiIV contained the Chitin_bind_1 domain (PF00187) and Glyco_hydro_19 domain (PF00182), MlChiV had the Glyco_hydro_18 domain (PF00704), MlChiID had the Chitin_bind_1 domain and Barwin domain (PF00967), while MlChiIC and MlHEL only contained the Glyco_hydro_19 domain and Barwin domain, respectively. Their subcellular location prediction showed that most of them were located in the vacuole except for MlChiV and MlHEL, which were located in the cell wall. 
The exon/intron analysis showed that except for MlChiIA and MlChiIC, which had two introns, all of the others only had one intron (Figure S4). The phylogenetic analysis of their full-length protein sequences showed that they could be divided into four groups (Figure S4), which was consistent with their exon/intron characteristics. Furthermore, the evolutionary relationships of the chitinases among different species were also explored. As shown in Figure 7, according to the evolutionary relationship of the chitinase gene family in mulberry [34] and Arabidopsis thaliana [35], these chitinases could be divided into five categories (Class I, II, III, IV, and V). The fifteen chitinases cloned in this study belong to Class I (MlChiIA, MlChiIC1, and MlChiIC2), Class II (MlChiID1, MlChiID2, MlHEL1, and MlHEL2), Class IV (MlChiIB, MlChiIV1, MlChiIV2, and MlChiIV3), and Class V (MlChiV1-1, MlChiV1-2, MlChiV2-1, and MlChiV2-2).

Then, the functional interactions of the nine chitinase proteins of $M$. laevigata were investigated in the STRING database with an Arabidopsis association model. The orthology analysis showed that MlChiIV1, MlChiIV2, MlChiIV3, and MlChiIB were homologous with the EP3 protein; MlChiIA, MlChiIC1, and MlChiIC2 were homologous with the HCHIB protein; MlChiV1-1, MlChiV1-2, MlChiV2-1, and MlChiV2-2 were homologous with the Chi protein; and MlHEL1, MlHEL2, MlChiID1, and MlChiID2 were homologous with the PR4 protein of Arabidopsis (Table S5). Finally, a total of 66 interaction protein pairs were predicted in Arabidopsis (Table S6, Figure S5). MlChiIA/MlChiIC and MlChiV had more interaction proteins than MlChiIV/MlChiIB and MlHEL/MlChiID, and these four group proteins also showed varying degrees of interaction with each other (Figure S5). Additionally, GO enrichment analysis showed that the predicted interaction proteins were significantly enriched in defense response, immune response, response to external stimulus, systemic acquired resistance, chitin binding, and chitinase activity (Table S7).

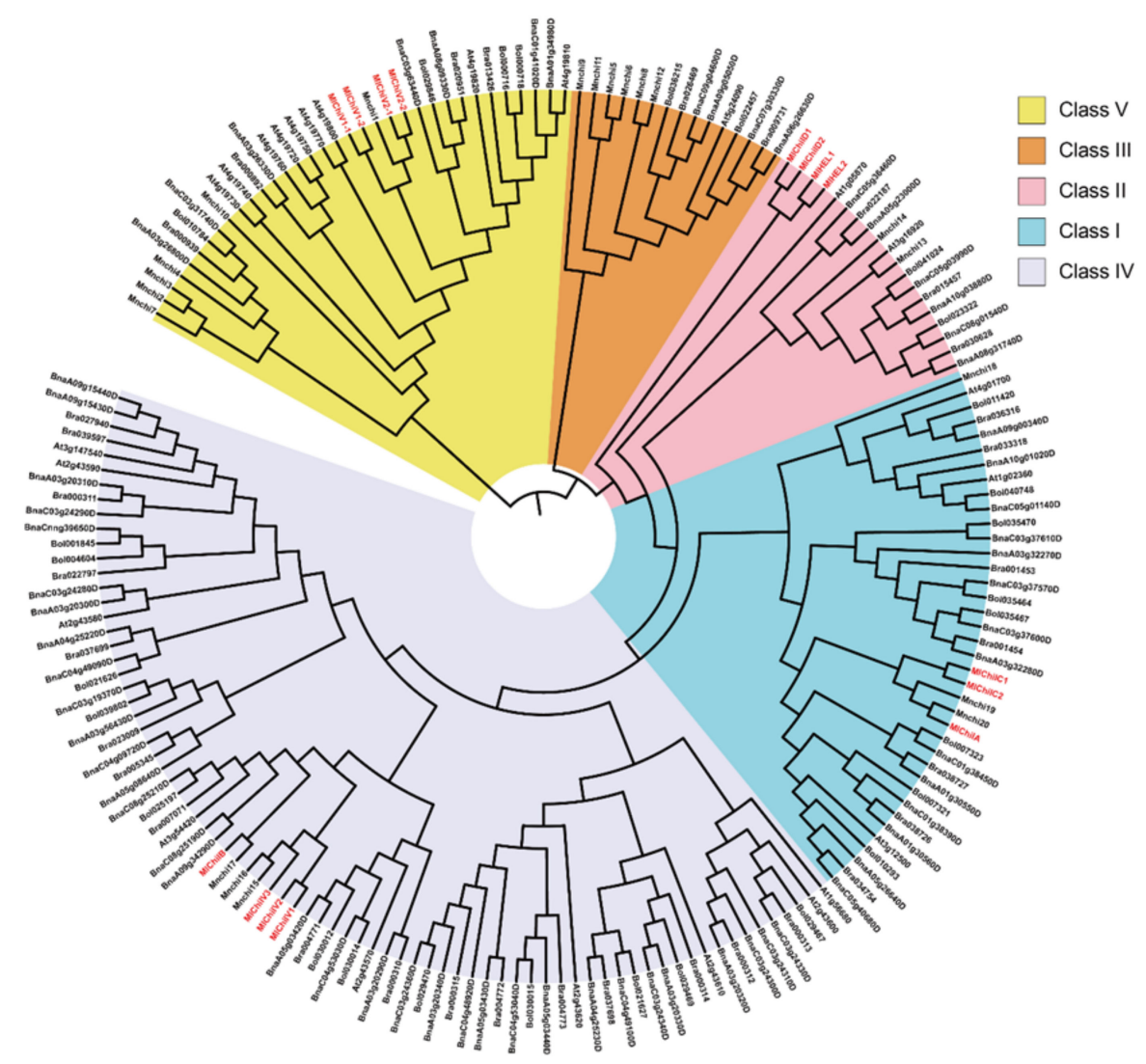

Figure 7. Phylogenetic relationship of the chitinases in different plants. The unrooted phylogenetic tree was constructed in MEGA6.0 using the NJ method with 1000 bootstrap replicates, and was visualized using the iTOL (https://itol.embl.de/) online tool. 
Table 3. Characteristics of the fifteen MlChi genes isolated from M. laevigata in this study.

\begin{tabular}{|c|c|c|c|c|c|c|c|c|c|c|c|}
\hline $\begin{array}{l}\text { Gene } \\
\text { name }\end{array}$ & Seq_ID & $\begin{array}{c}\text { gDNA } \\
\text { (bp) }\end{array}$ & $\begin{array}{l}\text { cDNA } \\
\text { (bp) }\end{array}$ & $\begin{array}{l}\text { ORF } \\
\text { (bp) }\end{array}$ & AA & SP & MW (kDa) & $p I$ & GRAVY & Domain & Subcellular localization \\
\hline MlChiIA & c46612 & 1997 & 1265 & 972 & 323 & $\mathrm{Y}$ & 34.95 & 6.84 & -0.37 & Chitin_bind_1 and Glyco_hydro_19 & vacuole \\
\hline MlChiIB & c34352 & 2167 & 1176 & 840 & 279 & $\mathrm{Y}$ & 30.39 & 4.63 & -0.32 & Chitin_bind_1 and Glyco_hydro_19 & vacuole \\
\hline MlChilC1 & c36015 & $\mathrm{N}$ & 1052 & 750 & 249 & $\mathrm{Y}$ & 27.36 & 5.57 & -0.36 & Glyco_hydro_19 & vacuole \\
\hline MlChiIC2 & c36015 & 1548 & 1039 & 750 & 249 & $\mathrm{Y}$ & 27.73 & 5.10 & -0.39 & Glyco_hydro_19 & vacuole \\
\hline MlChiID1 & c42529 & 1664 & 1052 & 633 & 210 & $\mathrm{Y}$ & 22.34 & 6.76 & -0.31 & Chitin_bind_1 and Barwin & vacuole \\
\hline MlChiID2 & c42529 & 1666 & 1054 & 636 & 211 & $\mathrm{Y}$ & 22.41 & 6.76 & -0.29 & Chitin_bind_1 and Barwin & vacuole \\
\hline MlHEL1 & c42297 & 1629 & 753 & 435 & 144 & $\mathrm{Y}$ & 15.78 & 6.79 & -0.20 & Barwin & cell wall \\
\hline MlHEL2 & c42297 & 1284 & 753 & 435 & 144 & $\mathrm{Y}$ & 15.75 & 6.04 & -0.21 & Barwin & cell wall \\
\hline MlChiV1-1 & c46798 & 3600 & 1307 & 1140 & 379 & $\mathrm{Y}$ & 42.15 & 4.96 & -0.22 & Glyco_hydro_18 & cell wall \\
\hline MlChiV1-2 & c46798 & 3603 & 1307 & 1140 & 379 & $\mathrm{Y}$ & 41.98 & 4.88 & -0.21 & Glyco_hydro_18 & cell wall \\
\hline MlChiV2-1 & c46798 & $\mathrm{N}$ & 1308 & 1173 & 390 & $\mathrm{Y}$ & 43.51 & 5.89 & -0.23 & Glyco_hydro_18 & cell wall \\
\hline MlChiV2-2 & c46798 & $\mathrm{N}$ & 1308 & 1173 & 390 & $\mathrm{Y}$ & 43.57 & 6.14 & -0.27 & Glyco_hydro_18 & cell wall \\
\hline MlChilV1 & c36895 & 1847 & 1329 & 822 & 273 & $\mathrm{Y}$ & 29.48 & 4.66 & -0.42 & Chitin_bind_1 and Glyco_hydro_19 & vacuole \\
\hline MlChilV2 & c36895 & 1849 & 1332 & 822 & 273 & Y & 29.48 & 4.62 & --0.42 & Chitin_bind_1 and Glyco_hydro_19 & vacuole \\
\hline MlChiIV3 & c36895 & 1848 & 1331 & 822 & 273 & Y & 29.39 & 4.61 & -0.38 & Chitin_bind_1 and Glyco_hydro_19 & vacuole \\
\hline
\end{tabular}

AA: Amino acid. SP: Signal peptide. MW: Molecular weight. $p I$ : Isoelectric point. GRAVY: Grand average of hydropathicity. 


\subsection{Prokaryotic Expression of the Ss-Inducible Chitinase Genes from M. laevigata}

The prokaryotic expression of the fifteen chitinase proteins from $M$. laevigata was performed in E. coli BL21 (DE 3.0). As shown in Figure S6, all of the recombinant proteins could be detected by the induction with $0.5 \mathrm{mM}$ IPTG, and the molecular weight of recombinant proteins was consistent with their theoretic values (Figure S6, Table 3). However, all the expressed proteins could only be detected in the precipitation fraction, not from the supernatant fraction, indicating that they are expressed as inclusion bodies. Considering that inclusion-body proteins were generally inactive, we did not carry out their purification and enzymatic activity detection.

\section{Discussions}

In this study, the transcriptome response characteristics of Ss-resistant mulberry stock M. laevigata after infection with $S s$ were revealed by RNA-seq. In total, 833 genes were differentially expressed after the infection. The DEGs analysis showed that most of them $(74.9 \%)$ were upregulated, which means that they took part in the M. laevigata's resistance to Ss. Consistent with the previous studies $[7,10,15,36,37]$, these DEGs were mainly involved in the oxidation-reduction process and metabolic process, which indicated that the DEGs involved in these biological processes might play special roles in response to the $S s$ invasion. These results provide the possibility for us to explore Ss-resistance candidate genes.

Transcriptional changes are a way for plants to respond to pathogen invasion, especially the expression of some defense genes that can be significantly induced in this process [36]. Hence, we selected 42 resistance-related genes as the major candidate genes from the upregulated DEGs. Functional annotation showed that most of them encoded enzymes, such as transferases, reductases, oxidases, and kinases. These enzymes have been reported to play important roles in the defense responses against pathogen infection, including $S s$, in different plants [10,38-40]. Some of them encode transcription factors, including MYB, NAC, ETR, TGA, and WRKY. Previous studies showed that these transcription factors played roles in the plant response to pathogen stress by controlling the synthesis of metabolites and affecting hormone signals, such as salicylic acid (SA), jasmonic acid (JA), and abscisic acid (ABA) signals [13,41,42]. For example, overexpression of BnWRKY33 enhanced the resistance of the transgenic rapeseed to $S$ s, which might be due to activation of the SA- and JA-mediated defense responses [29]. Additionally, there are seven genes $(c 36895, c 42297, c 46612, c 46798, c 34352$, c36015, and c42529) that encode chitinases among the candidate genes. Chitinases are often called pathogenesis-related proteins in plants, which play a dual role; they could inhibit pathogenic fungal growth by cell wall digestion, and could also release pathogen-borne elicitors to induce further defense reactions in the host plants [22,26]. They had been widely used to improve plant resistance to pathogens through biotechnology in various plants, such as tobacco [43], soybean [44], rapeseed [31,32], rice [45], and tea plant [46].

In order to further explore their roles in response to the infection of $S s$, we analyzed their expression characteristics in different organs of $M$. laevigata. In plants, $S$ s mainly causes lesions (rot symptoms) in nonflower organs such as stem, silique, pod, and fruit, though the flower petal is the organ for easiest infection. To our surprise, most of them (30/42) are specific to or mainly expressed in flowers, which is consistent with the fact that $S$ s mainly infects plants through flower organs, especially the petals [3]. This phenomenon also indicates that $S$ s-resistance genes could be induced by pathogen inoculation on ectopic organs. This might be one of the reasons for M. laevigata to resist Ss. Then, we also compared and analyzed the expression characteristics of the 42 candidate genes in response to $S s$ infection in different mulberry resistance stocks. As all of them were quickly induced in resistant stock M. laevigata, most of these genes were also induced by $S s$ infection in the two stocks with partial or low resistance, but the response paces were much slower and the extents were generally lower. In $M$. laevigata, most of these genes were significantly upregulated at $3 \mathrm{~h}$ after infection. In low-resistance stock $M$. alba cv. Zhenzhubai $(\mathrm{Ma})$ and partial-resistance stock M. alba $\times$ M. atropurpurea hybrid cv. Jialing $40(\mathrm{JL})$, they were significantly upregulated at $48 \mathrm{~h}$ after inoculation. Generally speaking, the difference in 
response to pathogen invasion and resistance often depends on the difference in time of recognition and activation of their own defense system of plants [21]. If plants can quickly identify the pathogens, transmit signals, and initiate defensive responses, then they will show resistance in their interactions with pathogens. Otherwise, the plants will be affected. These phenomena had been observed in different studies $[7,10]$, for example, Wu et al. found that the S-line rapeseed was more easily infected by $S s$ than the R-line, and the fold changes in many DEGs to the response to $S s$ inoculation were more dramatic in the R-line than in the S-line [2]. Therefore, the differences in the response of these candidate genes between $M$. laevigata and the two more-susceptible stocks are related to their resistance, which indicate that the differential expression of these candidate genes play an important role.

The genome of $S s$ contains a large number of different classes of virulence factors, including cell wall-degrading enzymes (CWDEs), signal cascade components, proteases and hydrolase, fungal nutrition and responding to environmental factors, reactive oxygen species (ROS) suppressors, and secreted proteins, which contributes to its wide range of infectivity and pathogenicity [23,47]. Therefore, it is necessary to fundamentally enhance its plant resistance. Among the 42 major candidates, chitinase genes of various types accounted for the largest group, so they were cloned and further studied. Cloning and functional investigation of the remaining candidate genes (e.g., the transcription factors) are also important for the resistance mechanism, which needs future study. Chitinase could degrade chitins, which are the primary structural components of the fungal cell walls, causing the cell walls' degradation and death of pathogens [22,26]. In our study, for the seven Ss-inducible chitinase genes, we found that all of them were specially expressed in leaves or flowers and were apparently induced by $S s$ infection. Additionally, they also showed a more dramatic increase in expression levels at the early stage of infection in M. laevigata than in the two more-susceptible stocks (Figure 6). Wu and collaborators also found that the difference in the fold changes in defense-related genes, such as chitinases, were much more dramatic than the RLKs, MAPK, and WRKY genes between the R- and $S$-lines in response to $S s$ [2]. These results suggest that chitinase plays a primary role in response to $S s$ infection. Therefore, we then focused on the isolation and sequence analysis of these $S$ s-induced chitinase genes. Our results showed that the $S$ s-induced chitinase genes isolated from M. laevigata had the typical chitinase characteristics, such as relatively small molecular weight, fewer introns, and conserved domains, mainly located in the secretion pathway [48]. These also indicated the conservation of their functions. It has been proved that chitinase genes could respond to the infection of various pathogens in different plants [49,50], and their overexpression could enhance the disease resistance of plants $[19,43]$. Therefore, the full-length gDNA and cDNA of the MlChi genes were cloned in our study, which provided a basis for further functional research. The interaction between plants and pathogens was a complex process, which required a series of regulatory networks to play a role [21,28]. By using Arabidopsis homologous genes for protein interaction network prediction, we found that these MlChi proteins could be divided into four categories, and they interacted with many defense response proteins (Figure S5). These not only further indicated that they were involved in Ss response, but also revealed that they might participate in the $M$. laevigata response to $S s$ through interaction with other defense proteins.

We also tried prokaryotic expression of Ss-inducible M. laevigata chitinase genes, but they all yielded inclusion bodies. As chitinase proteins are generally hydrophilic low-weight stable molecules, their inclusion body phenomenon might not be caused by physical characters. However, they contain higher cysteine residue frequencies than common proteins, which means that their correct folding might be more complicated. Under common prokaryotic expression conditions, cysteine-rich proteins might be prone to misfolding and then form inclusion bodies [51]. As this research involves multiple types and a number of chitinases, their proper expression and enzymatic characterization are not a simple issue, which will be further studied systematically.

In conclusion, in this study, we uncovered the transcriptome characteristics of the response to $S$ s in $M$. laevigata and initially screened out 42 candidate genes that might play central roles in response to $S s$ infection. These candidate genes not only had tissue-specific expression, but also 
had differential expression in response to $S s$ infection between resistant and susceptible mulberry stocks. The cloning and expression analysis of the $S s$-induced chitinase genes revealed their important functions in regulating the $M$. laevigata response to $S$ s infection. Our studies provided insights into the molecular basis of the resistance to $S s$ in M. laevigata, and the isolated MlChi genes could be potential candidate genes for the improving the $S s$ resistance of rapeseed and other crops through biotechnology.

\section{Materials and Methods}

\subsection{Plant Materials and Treatments}

In white rot disease, especially the Ss-resistance study, numerous papers have reported that in vitro inoculation on leaf or on stem is as effective as in vivo inoculation. In mulberry, white rot disease mainly causes heavy damage to fruits, the leaf can be infected, and light symptoms could be seen, while the stem of this tree can hardly be infected by $S$ s. As our purpose was to reveal the resistance mechanism and clone resistance genes, we chose mulberry leaf for in vitro inoculation. As mulberry trees are big and tall, and it is not easy to control and evenly treat under the open-air condition, we adopted the in vitro leaf inoculation method in this study. In early spring before the prevalence of natural Ss inoculation, young leaves were fetched for in vitro inoculation at room. Three mulberry varieties, including one wild resource $M$. laevigata (abbreviated as $\mathrm{Ml}$, resistant to $S s$ ) and two cultivated varieties, $M$. alba cv. Zhenzhubai (abbreviated as Ma, lowly resistant to $S$ s) and $M$. alba $\times M$. atropurpurea hybrid cv. Jialing 40 (abbreviated as JL, partially resistant to $S$ s), were used in this study. All of them were planted in the mulberry orchard of Southwest University, Chongqing, China. The newly fully developed young leaves of the three varieties from the healthy plants were collected for S. sclerotiorum inoculation. The inoculated leaves were cultured in the dark at room temperature. After 0,3, 9, and $48 \mathrm{~h}$ of inoculation, the leaf discs with a diameter of $2-3 \mathrm{~cm}$ around the inoculation point were collected as samples. Additionally, the leaves, flowers, stems, fruits, and roots of the Ml were also collected from the healthy plants as samples. Each sample had three biological replicates, and all of them were immediately frozen in liquid nitrogen and stored at $-80^{\circ} \mathrm{C}$ until further processing.

\subsection{RNA Extraction, and cDNA Library Preparation and Sequencing}

The total RNA of each sample was extracted using the EASYspin Plant RNA Kit (Biomed, Beijing, China) in accordance with the manufacturer's instruction. The quality of the isolated RNA was detected by $1 \%$ agarose gel electrophoresis, and the concentration was checked with the Qubit 2.0 Flurometer (Life Technologies, CA, USA). Equal amounts of RNA from the 3, 9, and $48 \mathrm{~h}$ of Ss-inoculation of $\mathrm{Ml}$ were mixed together as the treatment sample (ML_SS1), and the $0 \mathrm{~h}$ sample of $\mathrm{Ml}$ was the CK sample (ML_SSO); they were used for the construction of RNA-sequencing libraries. The sequencing libraries were generated using NEBNext Ultra ${ }^{\mathrm{TM}}$ RNA Library Prep Kit for Illumina (NEB, MA, USA) following the manufacturer's instructions, and index codes were added to attribute sequences to each sample. The RNA-seq was performed on the Illumina Hiseq 2000 platform with the Paired-end150 (PE150) strategy. The library construction and RNA-seq were performed by the commercial service of Novogene Co., Ltd. (Beijing, China).

\subsection{Data Processing, and Transcriptome Assembly and Annotation}

The raw reads of fastq format were first processed through in-house Perl scripts. Then, the clean reads were obtained by removing reads containing the adapter, the reads containing unknown bases $(>10 \%)$, and the low-quality reads (when the percentage of low-quality bases was over $50 \%$ in a read) from raw reads. At the same time, the Q20, Q30, GC-content, and sequence duplication level of the clean reads were calculated. Then, the clean reads were assembled using Trinity software [33] with min_kmer_cov set to 2 by default and all other parameters set to default to obtain the reference sequences, and the longest transcript of each gene was taken as the unigene for subsequent analysis. The gene function was annotated based on the following seven public databases: $\mathrm{Nr}$ (NCBI nonredundant protein 
sequences), Nt (NCBI nonredundant nucleotide sequences), Pfam (Protein family), KOG/COG (Clusters of Orthologous Groups of proteins), Swiss-Prot (A manually annotated and reviewed protein sequence database), KEGG (Kyoto Encyclopedia of Genes and Genomes database), and GO (Gene Ontology). The NCBI blast 2.2.28+ was used for functional annotation based on databases $\mathrm{Nr}$ (e-value $=1 \times 10^{-5}$ ), $\mathrm{Nt}\left(\mathrm{e}\right.$-value $\left.=1 \times 10^{-5}\right)$, Swiss-Prot $\left(\mathrm{e}-\mathrm{value}=1 \times 10^{-5}\right)$, and KOG/COG $\left(\mathrm{e}\right.$-value $\left.=1 \times 10^{-3}\right)$. The KAAS, HMMER3, and blast2go software were used for functional annotation based on databases KEGG $\left(\mathrm{e}\right.$-value $\left.=1 \times 10^{-10}\right)$, Pfam $(\mathrm{e}$-value $=0.01)$ and GO $\left(\mathrm{e}\right.$-value $\left.=1 \times 10^{-6}\right)$, respectively. These steps were performed by the commercial service of Novogene Co., Ltd. (Beijing, China) according to its pipelines standards.

\subsection{Differential Gene Expression, GO, and KEGG Enrichment Analysis}

The expression levels of genes were estimated using FPKM (Fragment Per Kilobase of Exon Model Per Million Mapped Reads) [52] by RSEM software [53]. Differential expression analysis between the two samples was performed using the DESeq R package (1.10.1). The resulting $p$ values were adjusted using the Benjamini and Hochberg's approach for controlling for the false discovery rate (FDR). Genes with an adjusted $p$-value $<0.05$ and fold change $(F C) \geq 2$ ( $\mid \log 2$ (fold change) $\mid \geq 1$ ) identified by DESeq were assigned as significantly differential expression. The GO enrichment analysis was implemented using the GOseq R packages-based Wallenius noncentral hyper-geometric distribution, and the KEGG pathways enrichment analysis was performed using the KOBAS software.

\subsection{Quantitative Real-Time PCR (qRT-PCR) Analysis}

For the qRT-PCR analysis, the reverse transcription of mRNAs using the total RNA as the start sample was performed using the PrimeScript ${ }^{\mathrm{TM}}$ RT Reagent Kit with gDNA Eraser (TaKaRa, Dalian, China) according to the manufacturer's instruction. The specific primers of each gene were designed using Vector NTI Advance 11.51 and Primer Premier 6 software. The qRT-PCR reactions were carried out using FastStart Essential DNA Green Master (Roche Diagnostics GmbH, Mannheim, Germany) on a CFX96 ${ }^{\mathrm{TM}}$ Real-Time System (Bio-Rad, Irvine, CA, USA) with three technical replicates according to the manufacturer's protocol. The 26SrRNA was used as the internal reference gene and the relative expression level of each gene was calculated using the following formula: $\mathrm{FC}=2^{-\Delta \Delta \mathrm{CT}}$ [54]. The Bio-Rad CFX Manager 3.0 software was used for data analysis. The primers used in qRT-PCR analysis are listed in Table S8.

\subsection{Cloning of cDNA and gDNA Sequences of Ss-Responsive Chitinase Genes from M. laevigata}

For the cloning of the chitinase genes from $M$. laevigata, the primers were designed based on comprehensive bioinformatics analysis, especially multi-alignment of the unigene sequences assembled in the M. laevigata transcriptome of this research, the M. notabilis genome references from the NCBI, and the in silico-cloned orthologous Morus EST and TSA tags from NCBI. The genomic DNA was isolated using the CTAB traditional method, and the CDNA of mixture-organ total RNA was obtained by reverse-transcription as described above. The PCR reactions were performed on the Veriti ${ }^{\mathrm{TM}} \mathrm{PCR}$ instrument (Applied Biosystems, Foster City CA, USA). The amplification products were purified via an EasyPure Quick Gel Extraction Kit (Transgen Biotech, Beijing, China) and cloned into a pMD19-T vector for sequencing. The primers used in this study are listed in Table S8.

\subsection{Sequence Characteristics, and Phylogenetic and Interaction Networks Analysis}

For the bioinformatics analysis of the cloned chitinase genes of $M$. laevigata, the Conserved Domain Database (CDD, https://www.ncbi.nlm.nih.gov/Structure/cdd/cdd.shtml) was used to verify their protein sequences. The physico-chemical characteristics, including molecular weight (MW), theoretical isoelectric point ( $p I)$, and grand average of hydropathicity (GRAVY), were calculated using the online Program tool of ExPASy (http://www.expasy.org/tools/). The signal peptide (SP) and transmembrane domain were predicted using the online tool SignalP 4.1 Server (http://www.cbs.dtu. 
dk/services/SignalP/) and TMpred Server (https://embnet.vital-it.ch/software/TMPRED_form.html), respectively. Two online tools Plant-mPLoc (http://www.csbio.sjtu.edu.cn/bioinf/plant-multi/) and TargetP1.1 (http://www.cbs.dtu.dk/services/TargetP/) were used to predict the subcellular localization. The encoded amino acid sequences of the chitinase genes from different plants were aligned using Clustal $\mathrm{X}$. Then, a phylogenetic tree based on the result of Clustal $\mathrm{W}$ protein alignments was constructed using the neighbor-joining (NJ) method in the software MEGA X with 1000 bootstrap replicates. Protein interaction network prediction was performed by STRING (https://string-db.org/) based on the homologous genes of Arabidopsis thaliana [55].

\subsection{Prokaryotic Expression}

The coding sequence of each gene with the signal peptide being removed was used as the template for primer design and vector construction. For MlChiIA, MlChiIB, MlchiIC1/2, MlchiID1/2, and MlHEL1/2, the fragment of each gene was cloned into the vector pET-28a (+), via the BamHI + HindIII restriction endonuclease recognition sites. For MlChiV1-1/2, MlChiV2-1/2, and MlChiIV1/2/3, the vectors were constructed by Bam HI + NotI and SacI + HindIII restriction enzymes, respectively. Then, the recombinant plasmid of each gene was transformed into E. coli strain BL21. The screened positive colonies of each gene were cultured in $\mathrm{LB}$ medium until the optical density $\left(\mathrm{OD}_{600}\right.$ value reached 0.6 at $37^{\circ} \mathrm{C}$ ); then, they were induced overnight by IPTG with a final concentration of $0.5 \mathrm{mM}$ at $28^{\circ} \mathrm{C}$. The recombinant $E$. coli cells were harvested by centrifugation from $5 \mathrm{~mL}$ culture medium after IPTG induction, and then they were resuspended with $1 \mathrm{~mL}$ Tris- $\mathrm{NaCl}(\mathrm{pH}=8.0)$. After centrifugation, the cells were resuspended again with $0.8 \mathrm{~mL}$ Tris- $\mathrm{NaCl}$ for ultrasonic crushing in an ice bath. The crushing mode was: Power, $300 \mathrm{~W}$; ultrasonic, $1 \mathrm{~s}$; interval, $3 \mathrm{~s}$; and ultrasonic time, $5 \mathrm{~min}$. After the samples were crushed, they were centrifuged at $4{ }^{\circ} \mathrm{C}$ and $12,000 \mathrm{rpm}$ for $10 \mathrm{~min}$ to collect the supernatant and precipitation. Then, they were mixed with $5 \times$ loading buffer and were boiled for $10 \mathrm{~min}$. The mixtures were centrifuged at 12,000 rpm for $5 \mathrm{~min}$, and a total of $15 \mu \mathrm{L}$ supernatant was analyzed by $12 \%$ SDS-PAGE. The primers used for vector construction are shown in Table S8.

\subsection{Data Availability}

The raw sequence data reported in this paper have been deposited in the Genome Sequence Archive [56] in National Genomics Data Center [57], Beijing Institute of Genomics (China National Center for Bioinformation), Chinese Academy of Sciences, under accession number CRA002928 that are publicly accessible at https://bigd.big.ac.cn/gsa. We are now submitting the sequences of the 42 unigenes and the cloned Ss-inducible chitinase genes to NCBI GenBank.

Supplementary Materials: Supplementary materials can be found at http://www.mdpi.com/1422-0067/21/21/ 8358/s1.

Author Contributions: Conceptualization, Y.C., H.J. and X.J.; methodology, H.J., X.S., X.J. and Y.C.; software, H.J., L.W., Y.X. and Y.C.; validation, H.J., X.S. and X.J.; formal analysis, H.J., X.S., X.J. and Y.C.; investigation, X.S., H.J., X.J., C.Z., C.Y., Y.D., X.L. (Xiaodan Liu), R.X., X.L. (Xiaomei Liu), J.J., L.T. and L.L.; resources, X.J. and Y.C.; data curation, H.J. and Y.C.; writing—original draft preparation, H.J.; writing-review and editing, Y.C.; visualization, H.J.; supervision, Y.C., L.W. and Y.X.; project administration, Y.C. and X.J.; funding acquisition, Y.C. All authors have read and agreed to the published version of the manuscript.

Funding: This research was funded by the Specialized Research Fund for the Doctoral Program of Higher Education (20130182110006), National Key R\&D Program of China (2016YFD0100506), National Natural Science Foundation of China (31871549 and 31171177), Young Eagles Program of Chongqing Municipal Commission of Education (CY200215), and Innovation and Entrepreneurship Training Program for College Students of Chongqing City (S202010635195) and Southwest University (X202010635608).

Acknowledgments: We thank the research team of Maode Yu from Southwest University for providing the mulberry plants for sampling.

Conflicts of Interest: The authors declare no conflict of interest. 


\section{Abbreviations}

$\begin{array}{ll}\text { SsSSVP1 } & \text { A small secreted virulence-related protein } 1 \text { in Sclerotinia scleroterum } \\ \text { QCR8 } & \text { Cytochrome b-c1 complex, subunit 8 protein } \\ \text { SsCP1 } & \text { Cerato-platanin protein } 1 \text { in Sclerotinia scleroterum } \\ \text { PR1 } & \text { Pathogenesis-related protein 1 } \\ \text { MaMLX-Q1 } & \text { Latex chitinase protein } 1 \text { isolated from Mulberry variety Qiangsang 1 (Q1) } \\ \text { BLAST } & \text { Basic Local Alignment Search Tool } \\ \text { IPTG } & \text { Isopropyl beta-D-thiogalactopyranoside }\end{array}$

\section{References}

1. Hegedus, D.D.; Rimmer, S.R. Sclerotinia sclerotiorum: When "to be or not to be" a pathogen? Fems Microbiol. Lett. 2005, 251, 177-184. [CrossRef]

2. Wu, J.; Zhao, Q.; Yang, Q.; Liu, H.; Li, Q.; Yi, X.; Cheng, Y.; Guo, L.; Fan, C.; Zhou, Y. Comparative transcriptomic analysis uncovers the complex genetic network for resistance to Sclerotinia sclerotiorum in Brassica napus. Sci. Rep. 2016, 6, 19007. [CrossRef] [PubMed]

3. Bolton, M.D.; Thomma, B.P.H.J.; Nelson, B.D. Sclerotinia sclerotiorum (Lib.) de Bary: Biology and molecular traits of a cosmopolitan pathogen. Mol. Plant Pathol. 2006, 7, 1-16. [CrossRef] [PubMed]

4. Alizadeh, N.; Babai-Ahary, A.; Assadi, Y.; Valizadeh, M.; Passebaneslam, B. The Effects of Sclerotinia Stem Rot of Oilseed Rape on the Production and Quality of Extracted Oil. J. Sci. Technol. Agric. Nat. Resour. 2006, 3, 485-495.

5. Boland, G.J.; Hall, R. Index of plant hosts of Sclerotinia sclerotiorum. Can. J. Plant Pathol. 1994, 16, 93-108. [CrossRef]

6. Cao, J.Y.; Xu, Y.P.; Zhao, L.; Li, S.S.; Cai, X.Z. Tight regulation of the interaction between Brassica napus and Sclerotinia sclerotiorumat the microRNA level. Plant Mol. Biol. 2016, 92, 39-55. [CrossRef] [PubMed]

7. Mei, J.; Ding, Y.; Li, Y.; Tong, C.; Du, H.; Yu, Y.; Wan, H.; Xiong, Q.; Yu, J.; Liu, S. Transcriptomic comparison between Brassica oleracea and rice (Oryza sativa) reveals diverse modulations on cell death in response to Sclerotinia sclerotiorum. Sci Rep. 2016, 6, 33706. [CrossRef]

8. Seifbarghi, S.; Borhan, M.H.; Wei, Y.; Coutu, C.; Robinson, S.J.; Hegedus, D.D. Changes in the Sclerotinia sclerotiorum transcriptome during infection of Brassica napus. BMC Genom. 2017, 18, 1-37. [CrossRef] [PubMed]

9. Monazzah, M.; Tahmasebi Enferadi, S.; Rabiei, Z. Enzymatic activities and pathogenesis-related genes expression in sunflower inbred lines affected by Sclerotinia sclerotiorum culture filtrate. J. Appl. Microbiol. 2018, 125, 227-242. [CrossRef]

10. Ranjan, A.; Westrick, N.M.; Jain, S.; Piotrowski, J.S.; Ranjan, M.; Kessens, R.; Stiegman, L.; Grau, C.R.; Conley, S.P.; Smith, D.L. Resistance against Sclerotinia sclerotiorum in soybean involves a reprogramming of the phenylpropanoid pathway and upregulation of anti-fungal activity targeting ergosterol biosynthesis. Plant Biotechnol. J. 2019, 17, 1567-1581. [CrossRef] [PubMed]

11. He, N.; Zhang, C.; Qi, X.; Zhao, S.; Tao, Y.; Yang, G.; Lee, T.; Wang, X.; Cai, Q.; Li, D. Draft genome sequence of the mulberry tree Morus notabilis. Nat. Commun. 2013, 4, 2445. [CrossRef]

12. Chang, L.Y.; Li, K.T.; Yang, W.J.; Chung, M.C.; Chang, J.C.; Chang, M.W. Ploidy level and their relationship with vegetative traits of mulberry (Morus spp.) species in Taiwan. Sci. Hortic. 2018, 235, 78-85. [CrossRef]

13. Liu, Y.; Ji, D.; Chen, J.; Lin, T.; Wei, J.; Zhu, Y.; Lv, Z. Overexpression of the mulberry latex gene MaMLX-Q1 enhances defense against Plutella xylostella in Arabidopsis thaliana. Arch. Biol. Sci. 2017, 69, 269-276. [CrossRef]

14. Yuan-Zhang, K.; Fu-An, W.U. A Review on Pathogens of Mulberry Fruit Sclerotiniosis and its Control Technology. Sci. Seric. 2012, 38, 1099-1104.

15. Bao, L.; Gao, H.; Zheng, Z.; Zhao, X.; Qian, Y. Integrated Transcriptomic and Un-Targeted Metabolomics Analysis Reveals Mulberry Fruit (Morus atropurpurea) in Response to Sclerotiniose Pathogen Ciboria shiraiana Infection. Int. J. Mol. Ences. 2020, 21, 1789. [CrossRef]

16. Hong, S.K.; Wan, G.K.; Sung, G.B.; Nam, S.H. Identification and distribution of two fungal species causing sclerotial disease on mulberry fruits in Korea. Mycobiology 2007, 35, 87-90. [CrossRef]

17. Sultana, R.; Kim, K. Bacillus thuringiensis C25 suppresses popcorn disease caused by Ciboria shiraianain mulberry (Morus australis L.). Biocontrol Sci. Technol. 2016, 26, 145-162. [CrossRef] 
18. Lu, R.; Jin, X.; Zhao, A.; Ji, J.; Liu, C.; Li, J.; Pu, L.; Lu, C.; Yu, M. Cross Infection, Biological Characteristics and Genetic Relationship between Pathogens of Hypertrophy Sorosis Sclerotenisis from Mulberry and Sclerotinia Stem Rot from Oilseed Rape. Acta Agron. Sin. 2015, 41, 42-48. [CrossRef]

19. Zh, Z.; Ya, Y.; Zh, M.; Re, J.; Re, X. Field Resistance Evaluation of 21 Fruit Mulberry Germplasm Resources against Mulberry Fruit Hypertrophic Sclerote Disease. Sci. Seric. 2015, 6, 1011-1016.

20. Tikader, A.; Kamble, C.K. Mulberry wild species in India and their use in crop improvement-A review. Aust. J. Crop Sci. 2008, 2, 64-72.

21. Dodds, P.N.; Rathjen, J.P. Plant immunity: Towards an integrated view of plant-pathogen interactions. Nat. Rev. Genet. 2010, 11, 539-548. [CrossRef]

22. Sharma, N.; Sharma, K.P.; Gaur, R.K.; Gupta, V.K. Role of Chitinase in Plant Defense. Asian J. Biochem. 2011, 6, 29-37. [CrossRef]

23. Xu, L.; Li, G.; Jiang, D.; Chen, W. Sclerotinia sclerotiorum: An Evaluation of Virulence Theories. Annu. Rev. Phytopathol. 2018, 56, 311-338. [CrossRef]

24. Lyu, X.; Shen, C.; Fu, Y.; Xie, J.; Jiang, D.; Li, G.; Cheng, J. A Small Secreted Virulence-Related Protein is Essential for the Necrotrophic Interactions of Sclerotinia sclerotiorum with its Host Plants. Plos Pathog. 2016, 12, 1005435. [CrossRef]

25. Yang, G.; Tang, L.; Gong, Y.; Xie, J.; Fu, Y. A cerato-platanin protein SsCP1 targets plant PR1 and contributes to virulence of Sclerotinia sclerotiorum. New Phytol. 2017, 217, 739-755. [CrossRef] [PubMed]

26. Hamid, R.; Khan, M.A.; Ahmad, M.; Ahmad, M.M.; Abdin, M.Z.; Musarrat, J.; Javed, S. Chitinases: An update. J. Pharm. Bioallied Sci. 2013, 5, 21-29.

27. Schlumbaum, A.; Mauch, F.; Vögeli, U.; Boller, T. Plant chitinases are important inhibitors of fungal growth. Nature 1986, 324, 365-367. [CrossRef]

28. Jones, J.D.G.; Dangl, J.L. The plant immune system. Nature 2006, 444, 323-329. [CrossRef]

29. Wang, Z.; Fang, H.; Chen, Y.; Chen, K.; Li, G.; Gu, S.; Tan, X. Overexpression of BnWRKY33 in oilseed rape enhances resistance to Sclerotinia sclerotiorum. Mol. Plant Pathol. 2014, 15, 677-689. [CrossRef] [PubMed]

30. Liu, Q.; Liu, Y.; Tang, Y.; Chen, J.; Ding, W. Overexpression of NtWRKY50 Increases Resistance to Ralstonia solanacearum and Alters Salicylic Acid and Jasmonic Acid Production in Tobacco. Front. Plant Sci. 2017, 8, 1710. [CrossRef]

31. Zarinpanjeh, N.; Motallebi, M.; Zamani, M.R.; Ziaei, M. Enhanced resistance to Sclerotinia sclerotiorumin Brassica napus by co-expression of defensin and chimeric chitinase genes. J. Appl. Genet. 2016, 57, 417-425. [CrossRef] [PubMed]

32. Ziaei, M.; Motallebi, M.; Zamani, M.R.; Panjeh, N.Z. Co-expression of chimeric chitinase and a polygalacturonase-inhibiting protein in transgenic canola (Brassica napus) confers enhanced resistance to Sclerotinia sclerotiorum. Biotechnol. Lett. 2016, 38, 1021-1032. [CrossRef]

33. Grabherr, M.G.; Haas, B.J.; Yassour, M.; Levin, J.Z.; Thompson, D.A.; Amit, I.; Adiconis, X.; Fan, L.; Raychowdhury, R.; Zeng, Q.; et al. Trinity: Reconstructing a full-length transcriptome without a genome from RNA-Seq data. Nat. Biotechnol. 2011, 29, 644-652. [CrossRef]

34. Wang, X.; He, N.; Zeng, Q.; Xiang, Z. Identification and expression analyses of chitinase genes in mulberry (Morus L.) plants. Plant Omics 2015, 8, 183-189.

35. Passarinho, P.A.; de Vries, S.C. Arabidopsis chitinases: A genomic survey. Arab. Book. 2002, 1, e0023. [CrossRef]

36. Joshi, R.K.; Megha, S.; Rahman, M.H.; Basu, U.; Kav, N.N.V. A global study of transcriptome dynamics in canola (Brassica napus L.) responsive to Sclerotinia sclerotiorum infection using RNA-Seq. Gene 2016, 590, 57-67. [CrossRef]

37. Dai, F.; Wang, Z.; Li, Z.; Luo, G.; Wang, Y.; Tang, C. Transcriptomic and proteomic analyses of mulberry (Morus atropurpurea) fruit response to Ciboria carunculoides. J. Proteom. 2019, 193, 142-153. [CrossRef]

38. Zhao, J.; Wang, J.; An, L.; Doerge, R.W.; Chen, Z.J.; Grau, C.R.; Osborn, M.T.C. Analysis of gene expression profiles in response to Sclerotinia sclerotiorum in Brassica napus. Planta 2007, 227, 13-24. [CrossRef]

39. Zhao, J.; Buchwaldt, L.; Rimmer, S.R.; Sharpe, A.; Mcgregor, L.; Bekkaoui, D.; Hegedus, D. Patterns of differential gene expression in Brassica napus cultivars infected with Sclerotinia sclerotiorum. Mol. Plant Pathol. 2009, 10, 635-649. [CrossRef]

40. Arfaoui, A.; Hadrami, A.E.; Daayf, F. Pre-treatment of soybean plants with calcium stimulates ROS responses and mitigates infection by Sclerotinia sclerotiorum. Plant Physiol. Biochem. 2017, 122, 121-128. [CrossRef] 
41. Li, J.; Brader, G.; Palva, E.T. The WRKY70 Transcription Factor: A Node of Convergence for Jasmonate-Mediated and Salicylate-Mediated Signals in Plant Defense. Plant Cell 2004, 16, 319-331. [CrossRef] [PubMed]

42. Kishikaboshi, M.; Seo, S.; Takahashi, A.; Hirochika, H. The MAMP-Responsive MYB Transcription Factors MYB30, MYB55 and MYB110 Activate the HCAA Synthesis Pathway and Enhance Immunity in Rice. Plant Cell Physiol. 2018, 59, 903-915. [CrossRef]

43. Navarro-González, S.S.; Ramírez-Trujillo, J.A.; Peña-Chora, G.; Gaytán, P.; Suárez-Rodríguez, R. Enhanced Tolerance against a Fungal Pathogen and Insect Resistance in Transgenic Tobacco Plants Overexpressing an Endochitinase Gene from Serratia marcescens. Int. J. Mol. Sci. 2019, 20, 3482. [CrossRef]

44. Hu, L.; Li, C.; Wang, X.; Zhang, F.; Ruan, X. Overexpression of a Chitinase Gene from Trichoderma asperellum Increases Disease Resistance in Transgenic Soybean. Appl. Biochem. Biotechnol. 2016, 180, 1542-1558.

45. Karmakar, S.; Molla, K.A.; Chanda, P.K.; Sarkar, S.N.; Datta, S.K.; Datta, K. Green tissue-specific co-expression ofchitinaseandoxalateoxidase 4genes in rice for enhanced resistance against sheath blight. Planta 2016, 243, 115-130. [CrossRef]

46. Singh, H.R.; Deka, M.; Das, S. Enhanced resistance to blister blight in transgenic tea (Camellia sinensis [L.] O. Kuntze) by overexpression of class I chitinase gene from potato (Solanum tuberosum). Funct. Integr. Genom. 2015, 15, 461-480. [CrossRef]

47. Amselem, J.; Cuomo, C.A.; Van Kan, J.A.L.; Viaud, M.; Benito, E.P.; Couloux, A.; Coutinho, P.M.; De Vries, R.P.; Dyer, P.S.; Fillinger, S. Genomic analysis of the necrotrophic fungal pathogens Sclerotinia sclerotiorum and Botrytis cinerea. Plos Genet. 2011, 7, e1002230. [CrossRef]

48. Chen, J.; Piao, Y.; Liu, Y.; Li, X.; Piao, Z. Genome-wide identification and expression analysis of chitinase gene family in Brassica rapa reveals its role in clubroot resistance. Plant Sci. 2018, 270, 257-267. [CrossRef]

49. Gerhardt, L.B.D.A.; Sachettomartins, G.; Contarini, M.G.; Sandroni, M.; Ferreira, R.P.; Lima, V.M.; Cordeiro, M.C.R.; De Oliveira, D.E.; Margispinheiro, M. Arabidopsis thaliana class IV chitinase is early induced during the interaction with Xanthomonas campestris. Febs. Lett. 1997, 419, 69-75. [CrossRef]

50. Li, W.; Faris, J.D.; Muthukrishnan, S.; Liu, D.; Chen, P.; Gill, B.S. Isolation and characterization of novel cDNA clones of acidic chitinases and $\beta$-1,3-glucanases from wheat spikes infected by Fusarium graminearum. Theor. Appl. Genet. 2001, 102, 353-362. [CrossRef]

51. Moghadam, M.; Ganji, A.; Varasteh, A.; Falak, R.; Sankian, M. Refolding process of cysteine-rich proteins: Chitinase as a model. Rep. Biochem. Mol. Biol. 2015, 4, 19.

52. Trapnell, C.; Williams, B.A.; Pertea, G.; Mortazavi, A.; Kwan, G.; Van Baren, M.J.; Salzberg, S.L.; Wold, B.J.; Pachter, L. Transcript assembly and quantification by RNA-Seq reveals unannotated transcripts and isoform switching during cell differentiation. Nat. Biotechnol. 2010, 28, 511-515. [CrossRef]

53. Li, B.; Dewey, C.N. RSEM: Accurate transcript quantification from RNA-Seq data with or without a reference genome. BMC Bioinform. 2011, 12, 323. [CrossRef]

54. Chao, Q.; Gao, Z.F.; Zhang, D.; Zhao, B.G.; Wang, B.C. The developmental dynamics of the Populus stem transcriptome. Plant Biotechnol. J. 2018, 17, 206-219. [CrossRef] [PubMed]

55. Szklarczyk, D.; Gable, A.L.; Lyon, D.; Junge, A.; Wyder, S.; Huerta-Cepas, J.; Simonovic, M.; Doncheva, N.T.; Morris, J.H.; Bork, P.; et al. STRING v11: Protein-protein association networks with increased coverage, supporting functional discovery in genome-wide experimental datasets. Nucleic Acids Res. 2019, 47, D607-D613. [CrossRef]

56. Wang, Y.; Song, F.; Zhu, J.; Zhang, S.; Yang, Y.; Chen, T.; Tang, B. GSA: Genome Sequence Archive. Genom. Proteom. Bioinform. 2017, 15, 14-18. [CrossRef]

57. National Genomics Data Center Members and Partners. Database Resources of the National Genomics Data Center in 2020. Nucleic Acids Res. 2020, 48, D24-D33.

Publisher's Note: MDPI stays neutral with regard to jurisdictional claims in published maps and institutional affiliations. 